\title{
Etude de la formation d'un lit torrentiel : Dune à forte pente et pavage
}

\author{
Vincent Koulinski* \\ CEMAGREF, Division Protection contre les Erosions \\ Philippe Bois \\ Professeur ENSHMG-INPG \\ Philippe Lefort \\ INPG Consultant \\ Maurice Meunier \\ CEMAGREF, Division Protection contre les Erosions
}

\section{Introduction}

Les écoulements à fort transport solide, autres que les laves torrentielles, qui se produisent dans les cours d'eau à forte pente ont été l'objet depuis une vingtaine d'années d'un certain nombre de travaux de base [1,2,3], essentiellement sur modèles réduits en canal étroit. Des synthèses de ces différents travaux apparaissent également, portant sur l'ensemble ou sur partie des variables qui constituent le charriage hyperconcentré $[4,5,6]$. Mais une caractéristique

(*) Actuellement Ingénieur Conseil, 541, Route de la Rosière, 73700 Bourg-St-Maurice. commune à ces différents travaux est qu'ils portent sur des essais en régime permanent uniforme, ce qui rend leurs résultats peut-être éloignés des conditions naturelles. On a vérifié, par exemple, que les valeurs de la capacité de transport obtenues à partir de ces travaux sont trop grandes par rapport à celles déduites d'observations in situ [7], et doivent donc être réduites pour être utilisées dans des aménagements [4].

Une cause importante d'écart provient de l'éventuelle indisponibilité des sédiments sur les versants ou dans le lit du torrent. On ne s'intéressera pas ici à cet aspect, mais au phénomène qui en découle, à savoir la variabilité des phénomènes torrentiels, à la fois dans le temps, avec

Stream bed structure study : steep slopes dune and pavement

Stream beds exhibit chaotic features which are hardly understandable whereas they reflect the stream behaviour. We carried out some small-scale experiments in the aim of understanding their formation. As a first step a very small-scale model allowed us to quantify locally bed load and particle grading. Two distinct phenomena appeared:

* The hyperconcentrated bed load transport for which bed load is governed by hydraulic conditions. In this case, the stream bed is relatively fine-grained and regular, the flow is rapid and the solid concentration is high. The particle grading - less important - increases the motion of big boulders.

* The ordinary bed load transport - close to the incipient motion threshold - for which the solid transport is quite variable and the Froude number varies around unity. Fine grains are easily moved which induces an armouring process.

As a second step, using a wide open channel, we showed that the bed load intensity decreases when the bed width is able to increase. Additionally, the different morphological structures tend to stop fine and coarse grain motions while intermediate grains are moved downstream. The irreversibility of the bifurcation between hyperconcentrated and ordinary bed load transport leads to a strong spatial heterogeneity between these two phenomena. This diversity can be seen by the appearance of large scale morphological structures : steep slopes dunes. 


\section{Liste des variables}

$D_{P . L} \quad$ Diamètre caractéristique du Lit correspondant à la probabilité $P$.

$D_{P . T}$ Diamètre caractéristique du matériau Transporté correspondant à la probabilité $P$.

$E \quad$ Epaisseur de la couche d'échange.

$Q_{l} \quad$ Débit liquide total.

$q_{l} \quad$ Débit liquide par unité de largeur.

$Q_{s} \quad$ Débit solide total.

$q_{s} \quad$ Débit solide par unité de largeur.

$\begin{array}{ll}q_{s}^{*} & \text { Débit solide unitaire adimensionnel. } \\ R & \text { Rayon hydraulique. }\end{array}$ $\sqrt{\frac{8}{f}}$ Coefficient de perte de charge de la formule de Darcy Weissbach.

notamment l'effet des débits moyens ou faibles de fin de crue qui permettent la formation du pavage, et dans l'espace pendant la crue (divagations incessantes des divers chenaux d'écoulement).

Pour bien observer des phénomènes, on s'est surtout intéressé au cas des fortes crues qui remanient complètement le lit alluvial, les crues faibles ayant des effets moins marqués mais plus facilement observables sur le terrain, car elles se produisent plus fréquemment. On va dans un premier temps décrire les phénomènes torrentiels en crue pour en déduire comment les simuler sur modèle réduit; on indiquera ensuite le protocole des expérimentations effectuées; on terminera par l'analyse des résultats.

\subsection{Schématisation des phénomènes torrentiels}

Si on veut simplifier le déroulement d'une crue telle qu'on peut la voir par simulation sur modèle physique, on peut isoler trois périodes :

- La période initiale de montée en puissance de la crue, où les phénomènes s'installent, soit lentement, soit rapidement, en fonction de la montée de la crue. Cette période ne nous intéressera pas ici.

- La période de crue proprement dite, pour laquelle on supposera que la fourniture de sédiments est suffisante pour permettre une pleine liberté aux changements qui, sur un bief alluvial non contraint donné, affectent en permanence le nombre et l'emplacement des chenaux partiels de l'écoulement d'une part, et les débits (liquides et solides) globaux et la pente globale d'autre part. Ces changements sont plus rapides que les variations des apports (solides et liquides) d'origine externe. Ils se produisent par succession de périodes d'accalmie, où le lit torrentiel et les écoulements semblent globalement en équilibre, avec seulement des mouvements de grains, nombreux mais apparemment individuels, suivies de changements rapides provoqués en un lieu limité par un phénomène typique du charriage hyperconcentré que nous appelons la dune à forte pente et sur lequel nous allons revenir.

- Une période de décrue, où les apports solides externes n'interviennent plus; la configuration des chenaux est alors figée, seuls les plus importants recoivent un écoulement liquide et l'équilibre solide - liquide de l'écoulement torrentiel s'y fait essentiellement à partir de ce qui est en place; lorsque les apports solides amont se tarissent, il se produit un processus du pavage par tri granulométrique du transport (la présence de gros éléments dans le lit facilite l'arrêt des grosses particules venant de l'amont).

\section{Dune à forte pente}

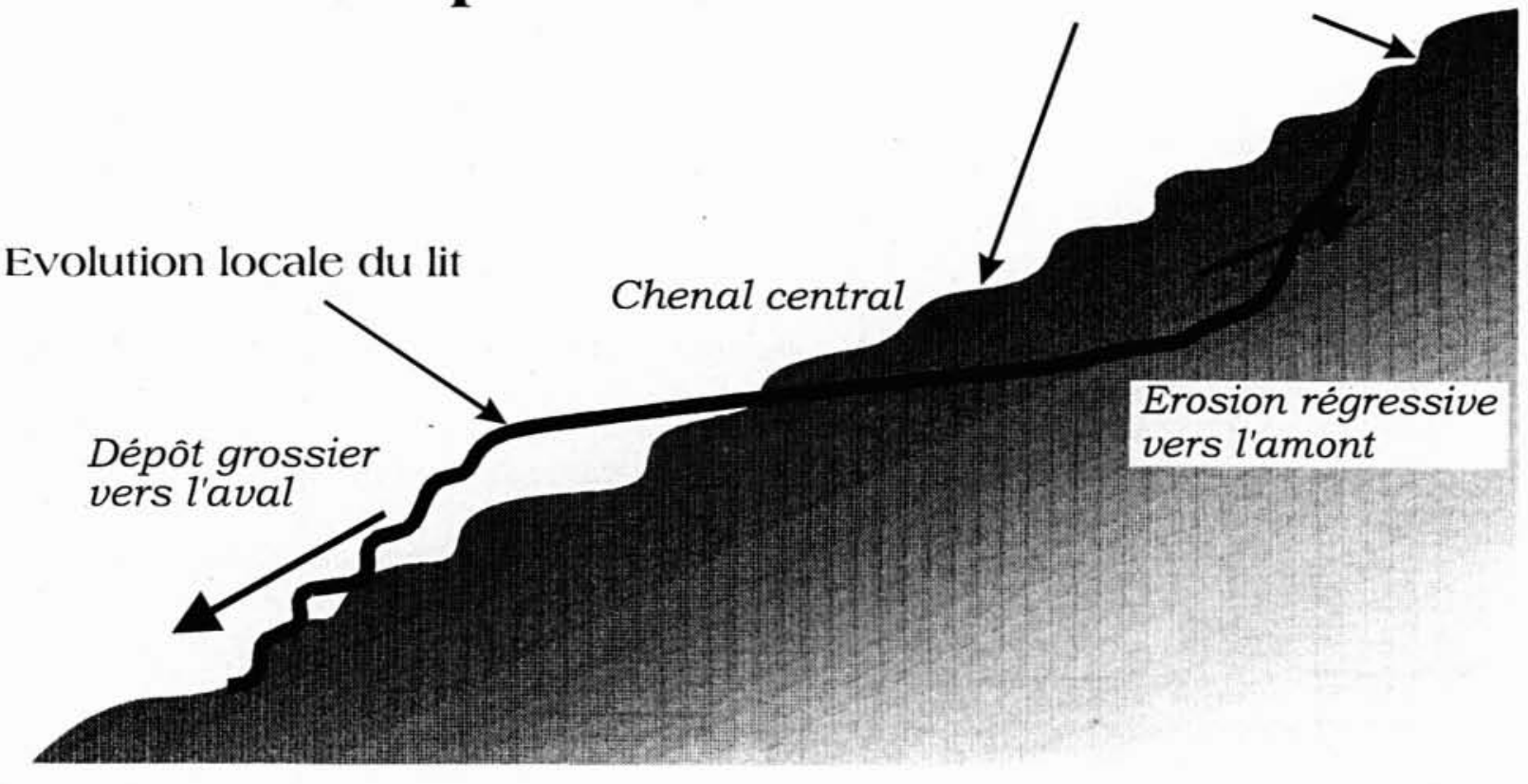

1. Schéma d'une nappe de charriage hyperconcentré. 


\subsection{La dune à forte pente}

Le schéma explicité ci-dessus simplifie évidemment ce qui se passe en réalité, avec l'influence des affluents, celle des berges qui peuvent contraindre l'écoulement, celle de la variabilité spatiale naturelle du profil en long, des apports latéraux, etc. Il correspond toutefois à ce qui peut être observé sur modèle réduit avec du matériau granulaire [13], observations qui permettent d'affirmer l'autonomie du phénomène primordial des changements rapides d'un lit torrentiel en crue : la dune à forte pente.

Celle-ci est un phénomène apparemment typique du transport solide des torrents, même si certaines structures similaires ont été notées sur les lits à gravier [8]. Pour les fortes pentes, elle a été observée par Mizurama [1] sous le nom de dune à forte pente. Le schéma de fonctionnement en est le suivant (fig. l) : elle est composée de trois parties: un chenal central bien marqué mais de faible pente, ayant un lit presque rectiligne et étroit, qui transporte un fort débit solide, avec des matériaux grossiers semblant rouler ou glisser sur un fond de matériaux plus fins, une niche amont en forme d'entonnoir, plus raide, qui collecte les apports amont d'un certain nombre de petits chenaux et peut reculer sous l'effet d'une érosion régressive provoquée par le fort transport du chenal, et enfin une zone de dépôt suélargissant à l'aval, qui est, elle aussi, à pente plus raide.

Une dune à forte pente s'installe lorsque les débits de plusieurs chenaux, en équilibre jusque là, se rassemblent dans un seul chenal, par suite de variations relativement lentes et de faible amplitude du modelé du lit alluvial, et donnent naissance à un écoulement liquide suffisant pour qu'un débit solide conséquent puisse être transporté. Un lessivage des éléments grossiers se produit alors et un lit fin à très forte capacité de transport s'installe avec érosion régressive et dépôt à l'aval. Ce processus se termine lorsque le bouleversement amont change les conditions d'alimentation, mais éventuellement aussi lorsque le dépôt s'est tellement étalé et épaissi qu'il change les conditions d'écoulement du chenal lui-même, qui est alors réparti en plusieurs chenaux à nouveau trop peu vigoureux pour générer un transport solide suffisant.

Ces "dunes à forte pente " ont un fonctionnement qui tranche nettement avec l'impression d'équilibre dynamique global que donne un écoulement torrentiel fort; il y a une accélération locale du processus de transport, de creusement et de dépôt qui fait facilement remarquer ce genre de processus quand il se produit sur le modèle réduit représentant le fonctionnement d'un bief alluvial non contraint. On concoit qu'il bouleverse en permanence la disposition des chenaux d'écoulement qui passent ainsi de manière erratique de la rive droite à la rive gauche, et vice-versa. Mais, comme il y a aussi interaction entre ces diffërentes dunes à forte pente, elles peuvent très bien s'amorcer sans se développer longtemps. Il n'est donc pas toujours évident de les reconnaitre.

L'observation de ces dunes à forte pente permet de penser que ce phénomène, relativement fugace, permet un déplacement de matériaux important, qui tranche avec le fonctionnement des autres chenaux. Leur variabilité explique donc sans problème, celle du transport solide en un point donné.

Dans la nature, on n'a guère la possibilité de constater la formation et l'évolution de ces dunes à forte pente pleinement développées. Par contre, on en voit les restes dans les cas favorables. Ce qui est observé plus facilement [13], ce sont des manifestations analogues avec des débits plus faibles ; étant plus fréquents, ces débits restructurent les dépôts de laves torrentielles ou d'un fort charriage hyperconcentré précédent et lui donnent l'apparence ordinaire des lits torrentiels.

\section{Approche expérimentale}

\subsection{Objectifs des essais}

L'objectif est d'étudier deux phénomènes importants de la dynamique torrentielle :

- pendant les crues, les dunes à forte pente constituent le phénomène prépondérant. On va étudier le fonctionnement du chenal central qui en est l'élément moteur ;

- en fin de crues, ou avec des crues médiocres (fonte des neiges), il se forme un pavage qui conditionne la réponse ultérieure du torrent aux crues. On va étudier son phénomène principal, le tri granulométrique.

De manière plus large, le souci est d'analyser le fonctionnement le plus fin possible de ces deux processus torrentiels, par obtention notamment de variables généralement inconnues : la granulométrie du transport solide. En effet si, en hydraulique fluviale, on assimile le plus souvent la granulométrie du transport solide à celle du lit, en hydraulique torrentielle, il y a une assez grande différence entre la granulométrie du matériau transporté pendant la crue et celle du lit torrentiel, avant, pendant et après la crue. Leurs liens sont relativement inconnus, les conséquences de leur confusion, également : par exemple, on s'interroge sur l'incertitude introduite par l'utilisation de la granulométrie du lit torrentiel dans les formules de transport solide, en lieu et place de la granulométrie du matériau transporté.

\subsection{Possibilité de représentation par modèle physique}

Ces deux phénomènes sont heureusement facilement accessibles à une étude sur modèle réduit, avec un canal étroit à berges fixes : on peut admettre en effet que la largeur du chenal d'écoulement est fixe et homogène dans les deux cas. Le premier cas correspond de plus aux nombreux essais de calcul de la capacité maximale de transport pour des chenaux de largeur constante, dont on a parlé précédemment. On en déduit d'ailleurs que le transport solide global sur un lit torrentiel alluvial ne peut qu'être inférieur à celui qui se produit dans le chenal d'une dune à forte pente; et qu'en conséquence, il est assez logique que les formules le surestiment.

\subsection{Protocole des essais}

Pour étudier ces deux phénomènes torrentiels, des essais sur modèle ont donc été conduits, sur canal de $10 \mathrm{~cm}$ de 


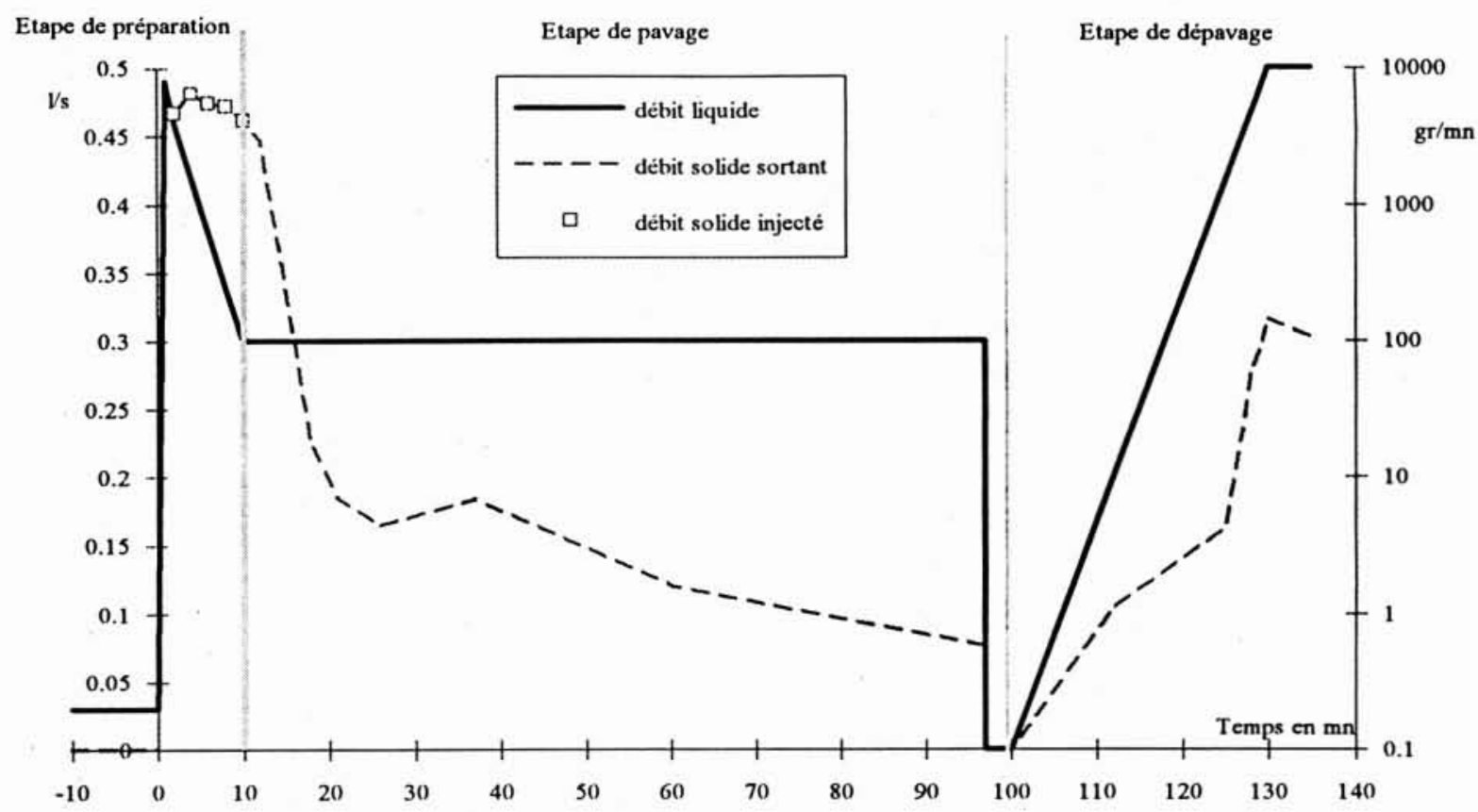

2 Déroulement en trois étapes d'un essai (essai 12).

large pour forcer le phénomène à rester unidimensionnel, puis de $60 \mathrm{~cm}$ de large pour une approche des phénomènes bi-dimensionnels. On a travaillé sur un lit à fond mobile avec un matériau de caractéristiques étalées $\left(D_{90}=\right.$ $9,2 \mathrm{~mm}, D_{50}=2,1 \mathrm{~mm}, D_{30}=1,1 \mathrm{~mm}$ ). Dans tous les cas, la pente de départ est $12 \%$. Le niveau aval est fixé par un seuil. Le protocole de l'essai est représenté sur la figure 2, avec trois étapes :

- $\mathbf{1}^{\text {re }}$ étape : le débit liquide décroît jusqu'à une valeur donnée. On injecte un débit solide variable de même granulométrie que celui du lit permettant d'obtenir les conditions hydrauliques les plus naturelles possibles et notamment la pente de départ souhaitée $(12 \%)$;

- $2^{\mathbf{e} e ́ t a p e ~: ~ u n ~ d e ́ b i t ~ l i q u i d e ~ c o n s t a n t ~ e s t ~ i n j e c t e ́ ~ s a n s ~ t r a n s-~}$ port solide. On arrête cette phase quand le débit solide sortant est devenu insignifiant. C'est la phase de formation et de consolidation du pavage ;

- $3^{\text {e }}$ étape : après une courte période d'arrêt du débit, nécessaire pour la mesure de la granulométrie, on fait croître le débit liquide jusqu'à obtention du dépavage.

Pendant toute la durée des essais, on effectue la mesure de la vitesse moyenne de l'écoulement par la méthode du sel [2], du profil en long, et du débit solide sortant total et de sa granulométrie. Le pas de temps de la mesure est adapté à l'importance du débit solide sortant. Pour accéder au cœur des relations entre l'écoulement et le lit torrentiel pendant la formation du pavage, on a essayé de travailler sur la longueur la plus faible possible de canal, $1 \mathrm{~m}$, et on a complété avec des essais à $2 \mathrm{~m}$ et $4 \mathrm{~m}$. Certains essais ont été doublés ou triplés pour tester la répétitivité des résultats. Les caractéristiques globales des essais se trouvent sur les tableaux $1 \& 2$ :
Tableau 1. Caractéristiques des essais en canal étroit

\begin{tabular}{|c|c|c|c|c|c|c|c||c|c|c|c|c|}
\cline { 2 - 13 } \multicolumn{1}{c|}{} & \multicolumn{4}{c||}{ Canal de deux mètres de long } & \multicolumn{5}{c|}{ Canal d'un mètre } \\
\hline numéro d'essai & 03 & 04 & 05 & 06 & 07 & 08 & 09 & 11 & 12 & 13 & 14 & 15 \\
\hline débit nominal (/s) & 0.3 & 0.3 & 0.25 & 0.25 & 0.25 & 0.2 & 0.2 & 0.25 & 0.3 & 0.2 & 0.25 & 0.2 \\
\hline volume injecté (1) & 30 & 26.4 & 19.5 & 20 & 25 & 22.5 & 23.5 & 23 & 27 & 17 & 19 & 22 \\
\hline durée totale (mn) & 60 & 90 & 110 & 82 & 76 & 120 & 135 & 180 & 120 & 120 & 120 & 120 \\
\hline pente initiale (\%) & 12.7 & 11.9 & 12.3 & 12.2 & 12.3 & 12.4 & 12.5 & 12.4 & 12.7 & 12.4 & 12.3 & 12.4 \\
\hline $\begin{array}{c}\text { pente après 10 mn } \\
(\%)\end{array}$ & 11.1 & 11.3 & 12.6 & 12.1 & 12.3 & 12.7 & 12.8 & 12 & 12.1 & 11.7 & 11.9 & 12.4 \\
\hline $\begin{array}{c}\text { pente du lit pavé } \\
(\%)\end{array}$ & 8.4 & 9.8 & 10.7 & 10.2 & 9.5 & 11.2 & 10.8 & 10 & 8.8 & 10.8 & 10.4 & 12.2 \\
\hline
\end{tabular}

Tableau 2. Caractéristiques des essais en canal de $60 \mathrm{~cm}$ de large.

\begin{tabular}{|l|c|c|c|c|c|c|}
\cline { 2 - 8 } \multicolumn{1}{c|}{} & $\begin{array}{l}\text { essai } \\
\text { prelimi. }\end{array}$ & \multicolumn{3}{c|}{ essais sans recyclage } & \multicolumn{2}{c|}{$\begin{array}{c}\text { essais avec } \\
\text { recyclage }\end{array}$} \\
\hline Numéro d'essai & $\mathbf{2 1}$ & $\mathbf{2 2}$ & $\mathbf{2 3}$ & $\mathbf{2 4}$ & $\mathbf{2 5}$ & $\mathbf{2 6}$ \\
\hline Débit nominal (1/s) & 0.4 & 0.3 & 0.35 & .4 & 0.3 & 0.4 \\
\hline Volume injecté (1) & 7.2 & 4 & 6 & 9.2 & 3.6 & \\
\hline Durée totale (mn) & & 159 & 50 & 122 & 110 & \\
\hline Pente initiale (\%) & 12.1 & 11.3 & 10.9 & 10.8 & 10.4 & \\
\hline
\end{tabular}

\section{Analyse globale des résultats obtenus (hors dépa- vage)}

\subsection{Examen de l'évolution du transport solide}

La courbe du débit solide au cours du temps (fig. 3). permet de constater une évolution similaire de tous les essais; on peut les séparer en trois phases:

— étape initiale avec apport solide amont. Le débit solide y est très important, de l'ordre de grandeur du débit solide injecté. On représente là l'écoulement du chenal central d'une nappe de charriage,

- formation du pavage: le transport solide qui ne 


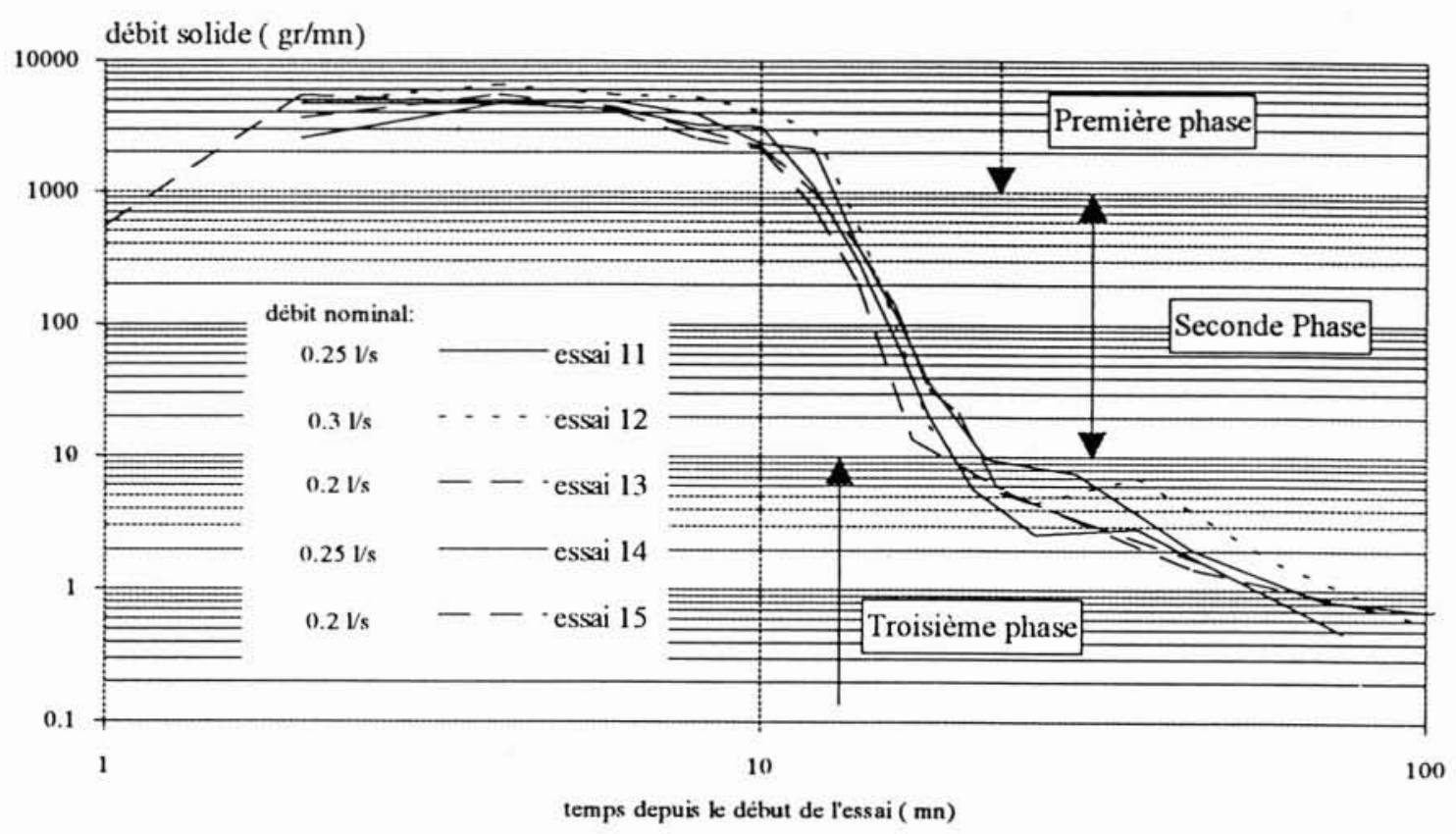

3. Evolution du débit solide dans le cas des essais en canal de $1 \mathrm{~m}$ de long.

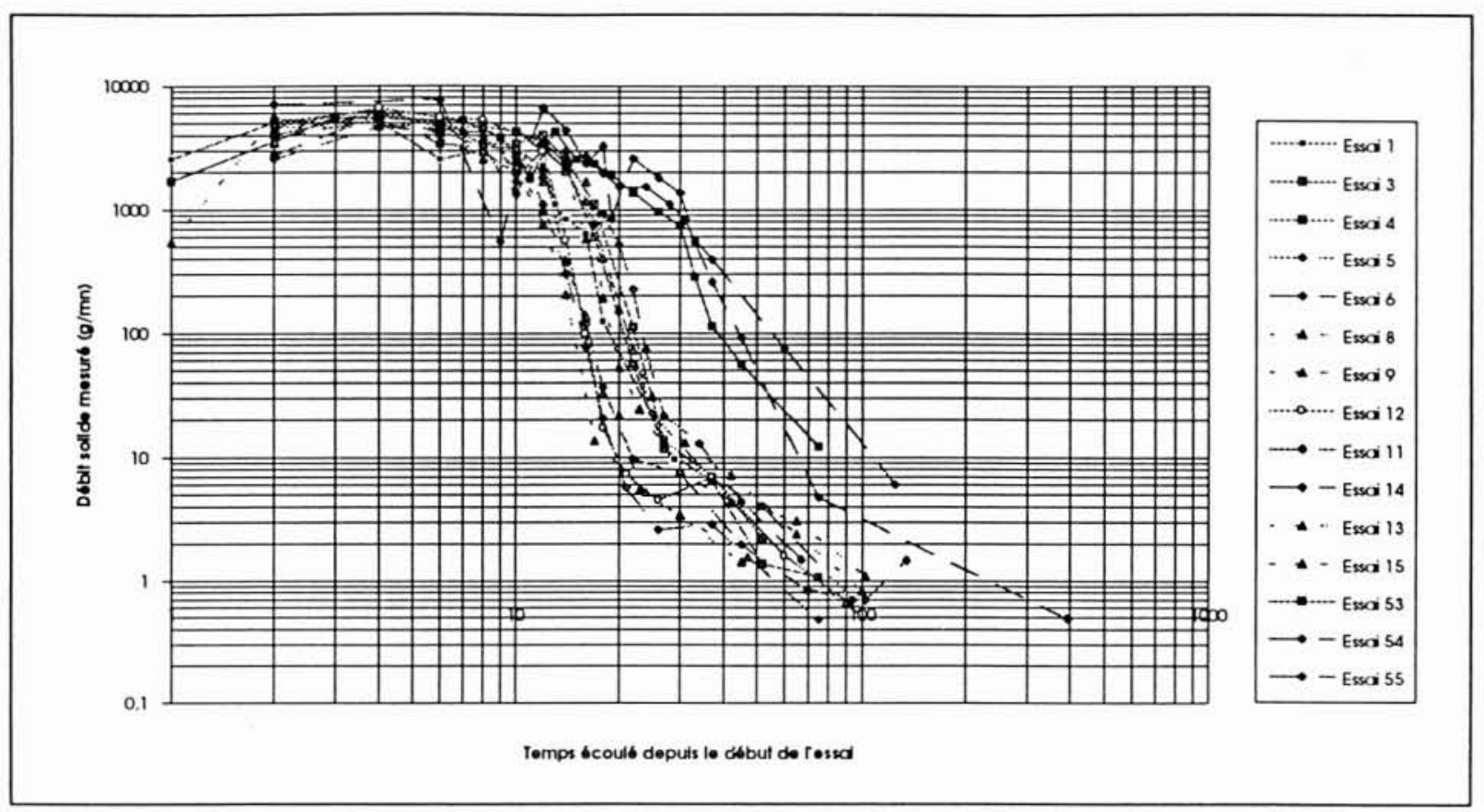

4. Effet de la longueur du canal lors de lévolution du débit solide en fonction du temps.

provient que du lit, décroit très vite, et on voit que corrélativement, la proportion de matériaux grossiers du lit augmente très vite,

- consolidation du lit: seuls les matériaux fins mal protégés peuvent s'échapper. Le débit solide est marginal, mais non nul.

La figure 4 montre les variations du débit solide pour l'ensemble des essais. Il apparait nettement que la décroissance dure d'autant plus que le canal est long. Il est possible de relier cette évolution à la propagation, dans le chenal, d'une zone de formation du pavage [12].

On comprend alors que, dans le cas des rivières où la fourniture en matériaux ne tarit jamais complètement, le pavage n'ait pas le temps d'atteindre le stade ultime comme lors de ces essais, mais reste dans un état intermédiaire de lit semi-pavé.

\subsection{Obtention de la granulométrie du lit en cours d'écou- lement}

Au début des essais, la granulométrie du lit est celle du matériau d'ensemble. Pendant les essais, elle ne peut être mesurée. Elle ne l'est qu'à la fin, à débit nul (obtention par la méthode des traversées sur photos [9]). On a obtenu celle 
du lit pendant l'écoulement à partir de celle du matériau transporté, qui est mesurée; pour cela, on a adopté une hypothèse couramment faite sur le fonctionnement de l'écoulement: celle de l'existence d'une couche active (ou couche de mélange, selon les auteurs), uniforme et homogène sur la longueur du canal. A partir d'une étude bibliographique $[10,11,12]$ sur les différentes hypothèses possibles et les modes de calcul de la granulométrie à partir de ce concept, on a adopté ici : $E=D_{90 . \mathrm{L}^{*}}\left(1.5+\beta q_{s^{*}}{ }^{2}\right)$, où $E$ est l'épaisseur de la couche active, $q_{s}{ }^{*}$ est le débit solide adimensionnel, et $\beta$ est un facteur multiplicatif (après calage, on a adopté $\beta=75$ ). Connaissant à tout moment les flux de transport solide pour chaque classe granulométrique, on en déduit la granulométrie du lit par un calcul régressif. Le résultat pour l'essai 3 se trouve sur la figure 5 .

La méthode peut être contrôlée en comparant la granulométrie initiale du lit obtenue par le calcul régressif et celle qui est mesurée (fig. 6).

On voit que la variabilité des mesures de la granulométrie de base est forte et qu'en conséquence les granulométries calculées sont encadrées par les valeurs mesurées. On en déduit que la confrontation est suffisamment bonne pour qu'on puisse admettre ce procédé de calcul et utiliser pour l'exploitation des résultats la granulométrie du lit ainsi calculée.

Ceci permet de voir l'évolution respective des deux granulométries au cours des essais, par exemple à travers celle du rapport $D_{50 . \mathrm{L}} / D_{50 . \mathrm{T}}$ indiqué sur la figure 7 .

\subsection{Confrontation aux formules d'hydraulique}

Cette confrontation peut être faite d'une part sur les formules de transport solide, d'autre part sur les formules de perte de charge.

\subsubsection{Formule de transport solide}

On teste ici la formule de Lefort [19] qui considère que les caractéristiques géométriques du lit sont un résultat du

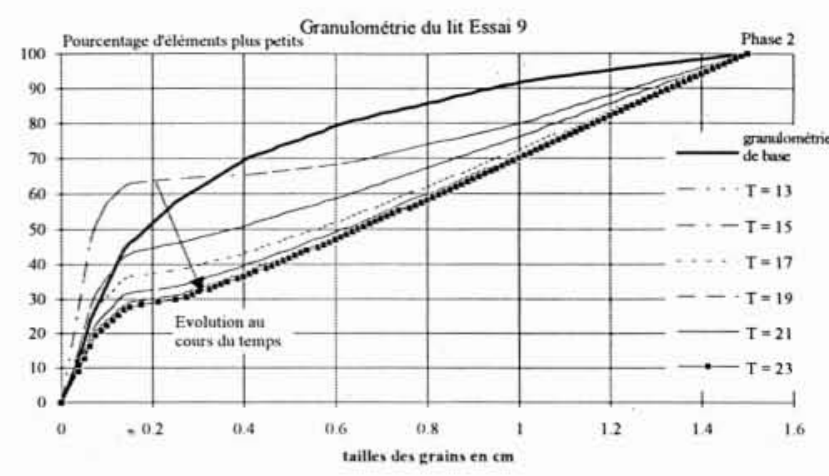

5. Evolution de la granulométrie du lit durant la phase 2 de l'essai 9 .

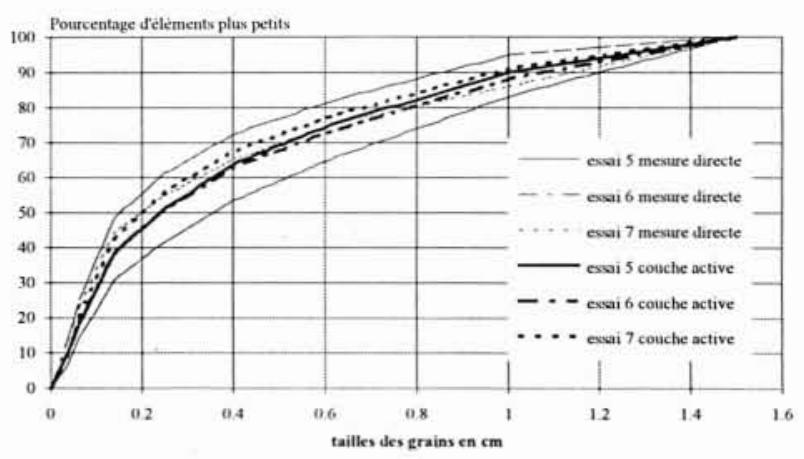

6. Comparaison des mesures directes de la granulométrie de base (traits fins) avec la granulométrie de base calculée par l'hypothèse de la couche active.

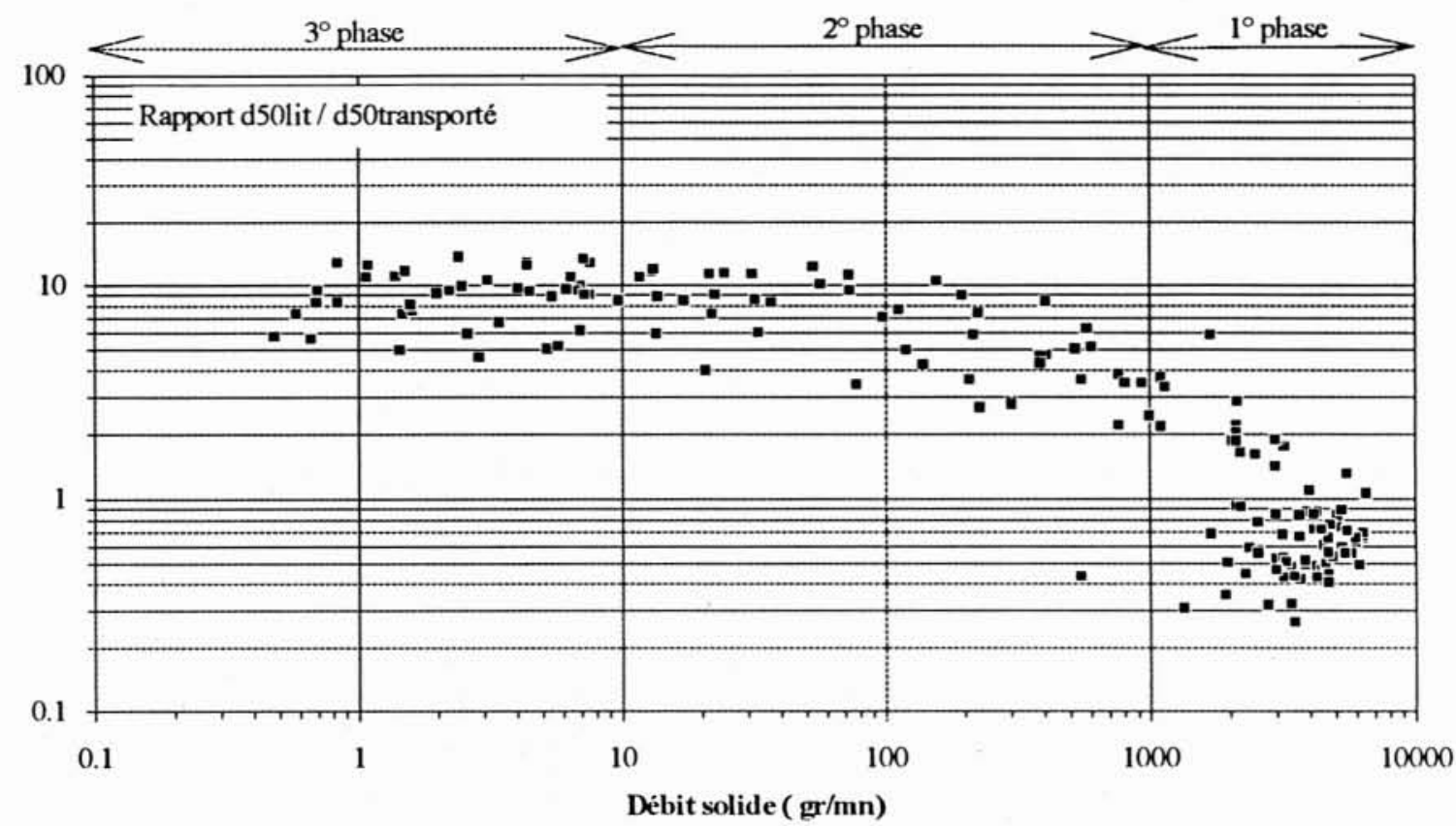

7. Comparaison de la gramulométrie des matériaux transportés et de celle du lit à travers le d50. 
transport solide. Le débit solide dépend alors uniquement du débit liquide total, ce qui permet d'éviter des interrogations sur les caractéristiques géométriques du lit en crue. Elle s'exprime, pour un matériau de densité 2,65, par:

$$
\frac{Q_{s}}{Q}=2.7\left(\frac{d_{90}}{d_{30}}\right)^{0.2} I^{1.5}\left(1-\left(\frac{Q_{l c}}{Q}\right)^{0.375}\right)
$$

avec

$$
\frac{Q_{\iota_{r}}}{\sqrt{g d_{m}^{5}}}=0.295 \times I^{-13 / 6}(1-1.2 I)^{8 / 3} .
$$

Ajustée sur 300 valeurs, correspondant à des conditions très différentes, elle fournit une valeur comprise entre la moitié ou le double des valeurs observées (pour $90 \%$ des points), ce qui est remarquable étant donnée la facilité d'emploi d'une telle formulation.

Cependant, il est nécessaire de choisir, dans l'utilisation des paramètres granulométriques, si l'on considère ceux du lit (l'arrachement d'un grain étant considéré comme le phénoméne principal) ou ceux du transport solide (le phénomène étant alors plutôt lié à l'écoulement). La figure 8 montre le résultat du calcul considérant les deux hypothèses (on notera que l'établissement de la formule est fait suivant la première hypothèse):

Comme on le voit, la formule ne donne de résultats valables que lors de la première phase, et ces résultats sont

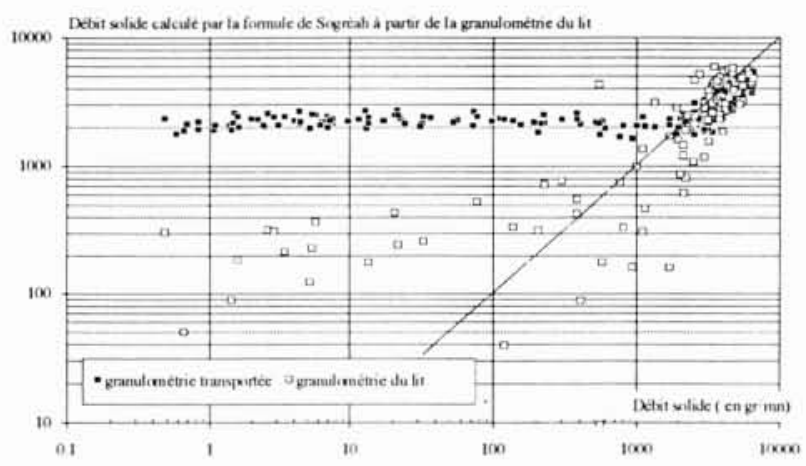

8. Confrontation du débit solide mesuré avec la formule de Lefort en considérant la granulométrie du lit ou la gramulométrie transportée.

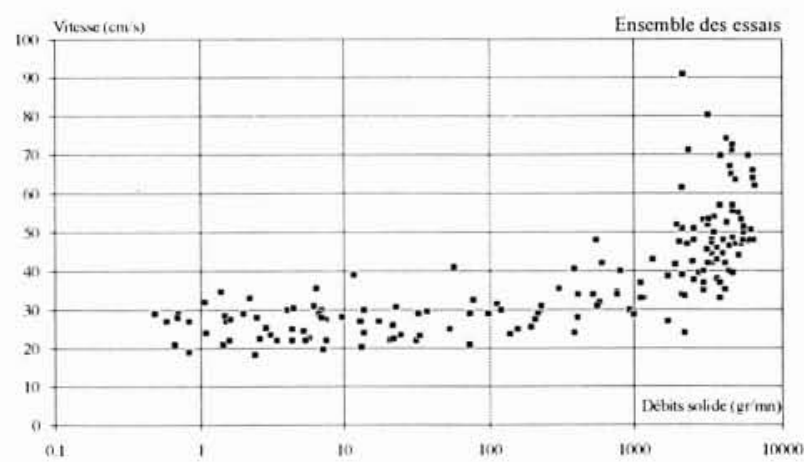

9. Vitesse mesurée en fonction du débit solide durant tous les essais de pavage. bons avec les deux types de granulométrie. Ceci est logique puisqu'en phase de fort charriage, notamment avec un apport de matériau à l'amont de granulométrie identique à celle du lit, elles sont assez proches (cf. fig. 7), le lit étant malgré tout nettement plus fin.

Mais la granulométrie du matériau transporté donne des résultats encore plus mauvais lorsque le pavage du lit commence. En effet, elle est d'autant plus fine que le lit est pavé, et donc que le débit solide est réduit. La formule est évidemment totalement incapable de prévoir une telle évolution, les paramètres globaux (pente, débit liquide) étant peu variables durant cette phase.

Finalement, il semble qu'il soit préférable d'utiliser la granulométrie du lit afin d'étendre un peu le domaine de validité de la formule. Cependant, il reste illusoire d'appliquer cette formule avec succès lorsque l'on se rapproche du seuil de début de transport dans le cas d'une granulométrie étendue.

\subsubsection{Formules de perte de charge}

A forte pente, les formules de perte de charge usuelles (Manning-Strickler) sont considérées comme inopérantes [4], ce qui peut être facilement vérifié ici. Le coefficient de rugosité $K$ reste à peu près constant $(K=16$ à 19) et ne correspond pas aux valeurs obtenues par la formule $K=26 d_{90 L}^{-1 / 6}$. D'autres formules ont été proposées, basées souvent sur la submersion relative $(R / D)$, ou sur la pente, ou sur l'influence du transport solide, lorsqu'il est suffisamment important pour intervenir dans la hauteur d'écoulement.

La figure suivante montre l'évolution de la vitesse en fonction du débit solide :

Il apparaît deux comportements distincts :

- Pour un fort transport solide (plus de $1000 \mathrm{~g} / \mathrm{min}$ ), le lit est très fin : sa contribution aux pertes de charge est alors considérablement réduite et l'écoulement est freiné essentiellement par les matériaux transportés. Mais, globalement, les pertes de charge sont quand même réduites, et lorsque le débit solide est important, l'écoulement est très rapide, le nombre de Froude dépassant largement l'unité (voir fig. 10). Cette phase est caractérisée par de très fortes variations de vitesse sans doute liées à celles du lit et du transport solide.

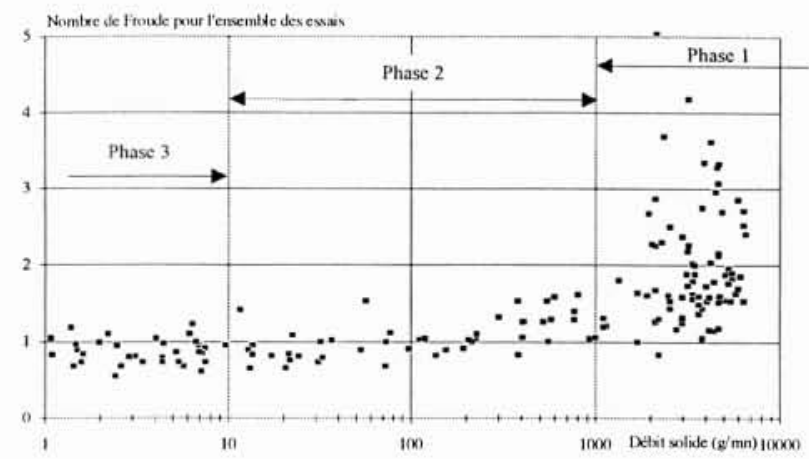

10. Nombre de Froude durant les essais. 
- Lorsque le lit est pavé, les pertes de charge sont causées par les gros éléments (la submersion relative est alors inférieure à l'unité) et surtout par les formes du lit (marches d'escalier en particulier). Le nombre de Froude moyen est alors nettement plus réduit et se situe généralement légèrement en dessous de l'unité.

La figure 10 montre l'évolution du nombre de Froude durant les essais :

Les formules classiques sont incapables de rendre compte de cette dualité. A titre d'exemple, on confronte sur la figure 11 les valeurs du coefficient de perte de charge $\sqrt{\frac{8}{\mathrm{f}}}$ d'une part calculées à partir des mesures, d'autre part prédéterminées avec la formule de Smart et Jaeggi [2].
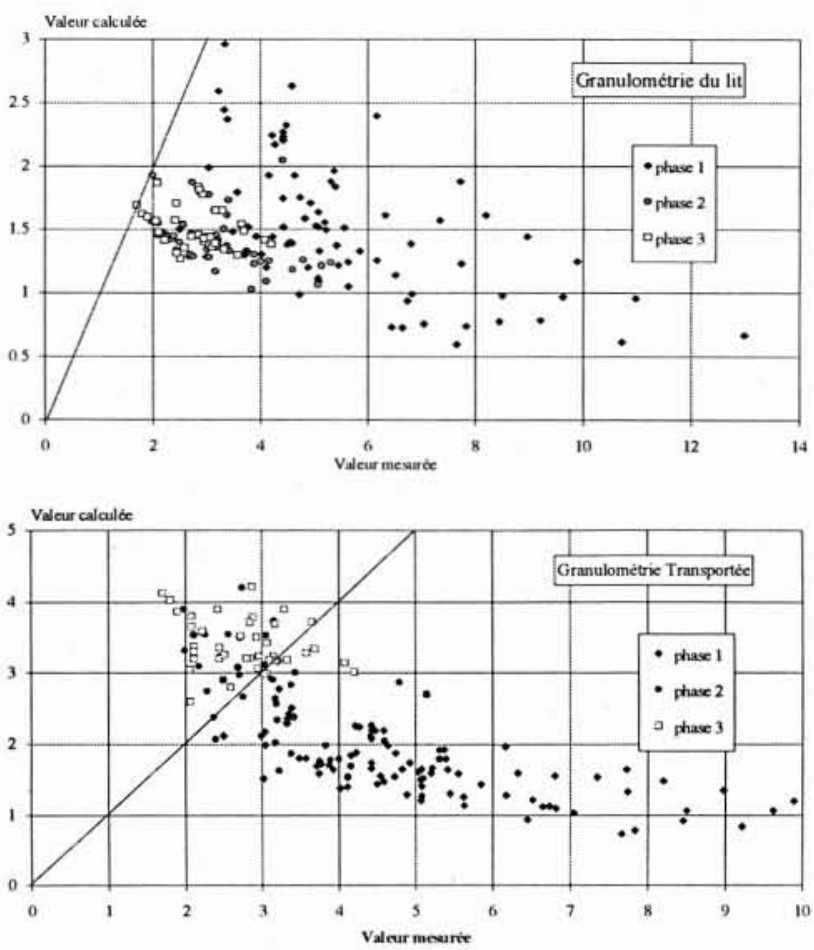

11. Valeurs de $\sqrt{\frac{8}{f}}$ calculée avec la formule de Smart \& Jaeggi en fonction de la granulométrie du lit ou de celle du transport solide.

\subsection{Tri granulométrique}

\subsubsection{Répartition en classes}

La différence de comportement des grains selon leur taille oblige à séparer la courbe granulométrique en classes. On en prend souvent un nombre important (10 ou 12) pour affiner la description du phénomène à représenter. Si cela ne pose pas de problème pour la modélisation mathématique, cela complique la représentation visuelle qui seule permet d'accéder au fonctionnement intime du phénomène. Aussi, pour " découvrir » le phénomène, nous avons travaillé avec 3 classes granulométriques seulement, les matériaux fins $(100 \mu \mathrm{m}-630 \mu \mathrm{m})$, moyens $(0,63 \mathrm{~mm}$ $2,5 \mathrm{~mm})$, et grossiers $(2,5 \mathrm{~mm}-15 \mathrm{~mm})$.

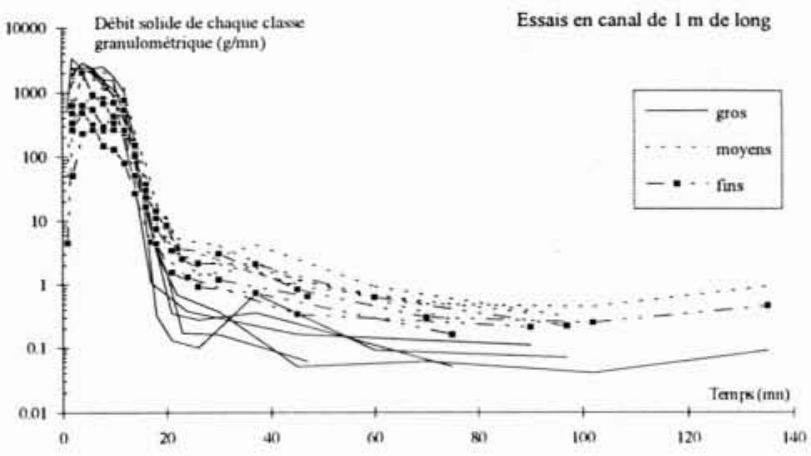

12. Débit solide en fonction du temps pour chaque classe granulométrique. Essai en canal de $1 \mathrm{~m}$.

\subsubsection{Evolution du débit solide par classes granulométriques}

La courbe 12 montre l'évolution au cours du temps des trois débits solides correspondant à ces trois classes granulométriques.

Comme le montre cette figure, la variation du débit solide au cours des essais est telle que les écarts de comportement entre les différentes classes sont gommés par l'amplitude des débits solides (le graphique couvre un rapport de 1 à 1000000 !).

Il a donc été nécessaire, pour mieux percevoir les évolutions granulométriques, de définir un paramètre comparant la mobilité d'une classe à sa fraction dans le lit : c'est l'indice de mobilité relative qui est abordé au paragraphe suivant.

\subsubsection{Variation respective de mobilité de chaque classe}

$\mathrm{Si}$ on appelle indice de mobilité relative d'une classe, le rapport de ses fréquences à un moment donné, respectivement dans le matériau transporté et dans la couche d'échange, on peut tracer l'évolution de cet indice au cours du temps (fig. 13).

La courbe montre que l'indice des matériaux grossiers se distingue des deux autres:

- Pendant la première phase (charriage hyperconcentré), il est supérieur à l'unité, ce qui avait déjà été noté [1] et correspond bien à l'observation des gros éléments roulant relativement facilement sur un tapis d'éléments fins dans le chenal d'une nappe de charriage hyperconcentré.

- Pendant la deuxième phase (formation du pavage), cet indice décroît fortement au contraire des indices des deux autres classes.

- Enfin, il reste constant pendant la phase de lessivage.

La forte décroissance de l'indice de mobilité relative des éléments grossiers pendant la phase de formation du pavage traduit très bien la dynamique du tri granulométrique pendant cette phase : le déplacement par paquets des gros éléments pendant la phase initiale devient plus difficile avec le débit de formation du pavage, trop faible, et certains de ces paquets s'arrêtent, causant une obstruction et augmentant alors la possibilité de s'arrêter en butée pour d'autres gros éléments. Le nombre de gros éléments dans le lit augmente pendant que celui dans le transport diminue. 


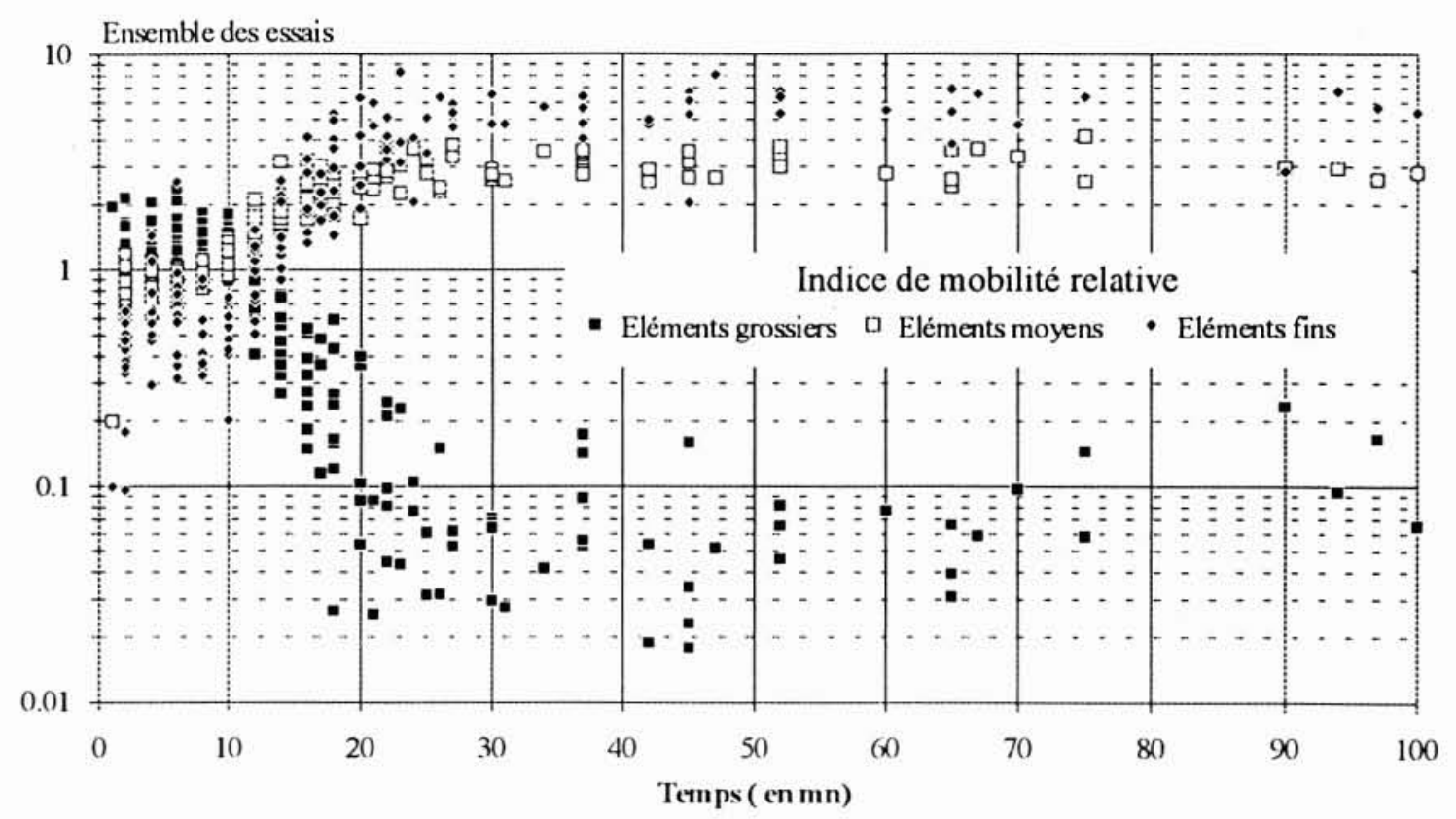

13. Evolution de l'indice de mobilité relative en fonction du temps pour l'ensemble des essais.

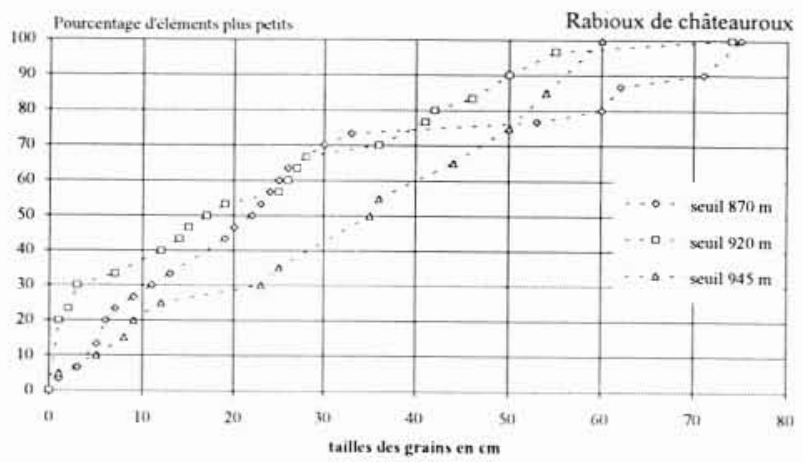

14. Courbes granulométriques sur le Rabioux de Châteauroux.

Ce processus d'arrêt des paquets de gros éléments fonctionne également avec des conditions hydrauliques plus vigoureuses; mais il se produit inévitablement quelques instants plus tard une déstabilisation de cet amas par érosion latérale ou régressive. Pour ces phénomènes torrentiels, le comportement des grains ne peut être considéré comme individuel, ce qui rend évidemment plus difficile l'élaboration d'un modèle théorique.

Il est intéressant de noter que l'évolution de la courbe granulométrique au cours du temps, la conduit ả se « linéariser " (fig. 14), ce qui pourrait presque servir de méthode de reconnaissance de l'état de surface d'un lit torrentiel, comme on peut le voir avec la courbe granulométrique des seuils sur le torrent du Rabioux (de Châteauroux).

\subsubsection{Etude des dépendances entre classes granulométriques}

La méthode usuelle pour tenir compte d'une différenciation des classes granulométriques consiste à utiliser un facteur de masquage, qui modifie soit la loi de transport solide [14], soit le seuil de début de transport [15, 16, 17, 18]. Ce facteur correctif dépend la plupart du temps du facteur $\left(D_{i} / D_{50}\right)$ et la forme de la fonction correctrice ainsi que les valeurs des paramètres de cette fonction sont obtenues par des considérations semi théoriques et semi expérimentales.

Cependant, les observations précédentes ont montré que la mobilité relative des classes granulométriques était très variable en fonction des conditions hydrauliques. Ainsi, l'objectif de cette démarche est d'éclairer le phénomène physique et de comprendre les relations qui relient les classes granulométriques du matériau du lit et du matériau transporté pendant les trois phases des essais.

Si l'on reconsidère l'évolution de l'indice de mobilité relative en fonction de la fraction d'éléments grossiers dans le lit, la décomposition en 3 phases apparaît nettement :

- Dans la phase de fort débit solide (fig. 15), il y a une forte indépendance entre le lit et le transport solide et il apparaît une dispersion importante.

Cependant, il n'y a pas de différence très marquée entre les classes, ni dans le lit ni dans le transport solide, même si les éléments sont d'autant plus mobiles qu'ils sont plus gros. Les matériaux grossiers et les matériaux de taille moyenne ont un comportement globalement similaire alors que les éléments fins apparaissent moins mobiles, et ont un comportement plus aléatoire.

- Au contraire, lors de la formation du pavage (fig. 16), les comportements diffèrent nettement d'une classe à l'autre :

Dans ce cas, les éléments grossiers perdent leur mobilité et encombrent le lit alors que les autres sont d'autant plus mobiles - et rares dans le lit - qu'ils sont fins.

- Enfin, le lessivage du lit (fig. 17) montre que l'on atteint un état stable, la dispersion des points étant nettement plus réduite à chaque essai, et qui correspond au lit pavé (au moins dans le cadre de ces essais).

La figure 18 , en regroupant les trois phases, montre la remarquable homogénéité des comportements des classes 


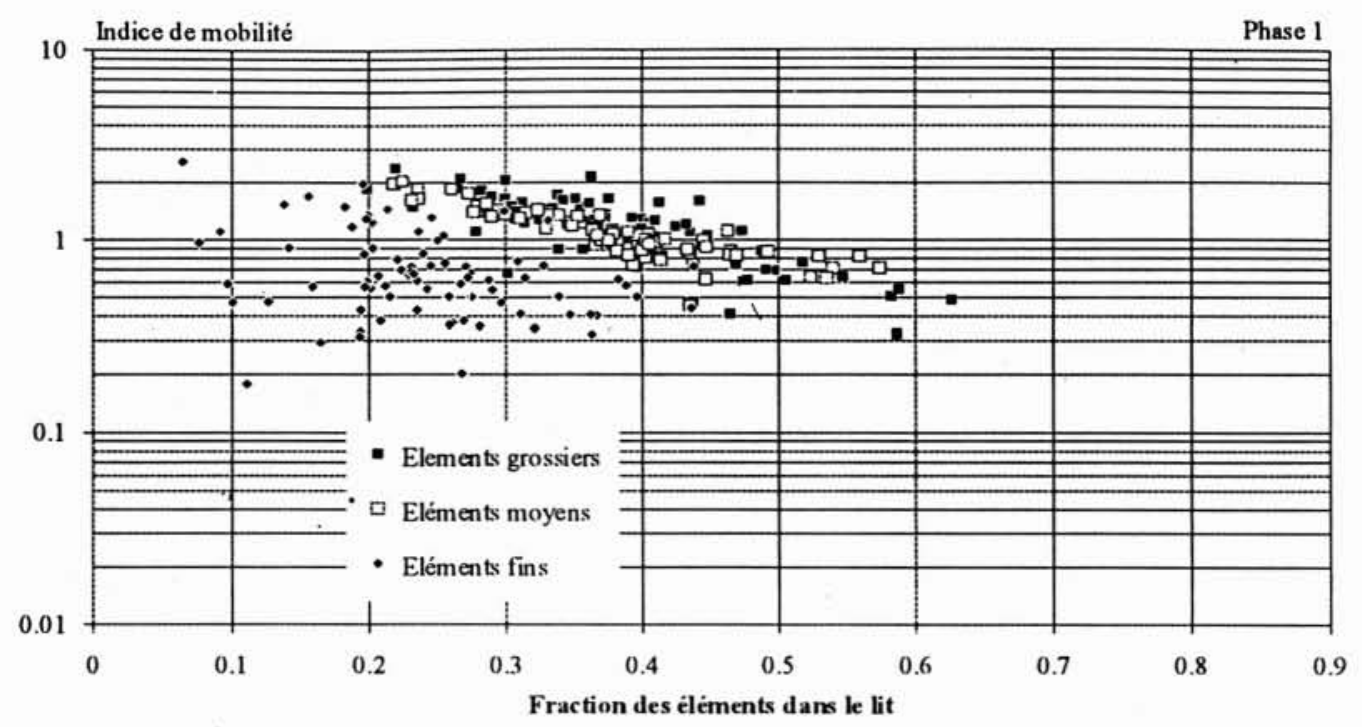

15. Indice de mobilité des éléments en fonction de leur fraction dans le lit durant la première phase.

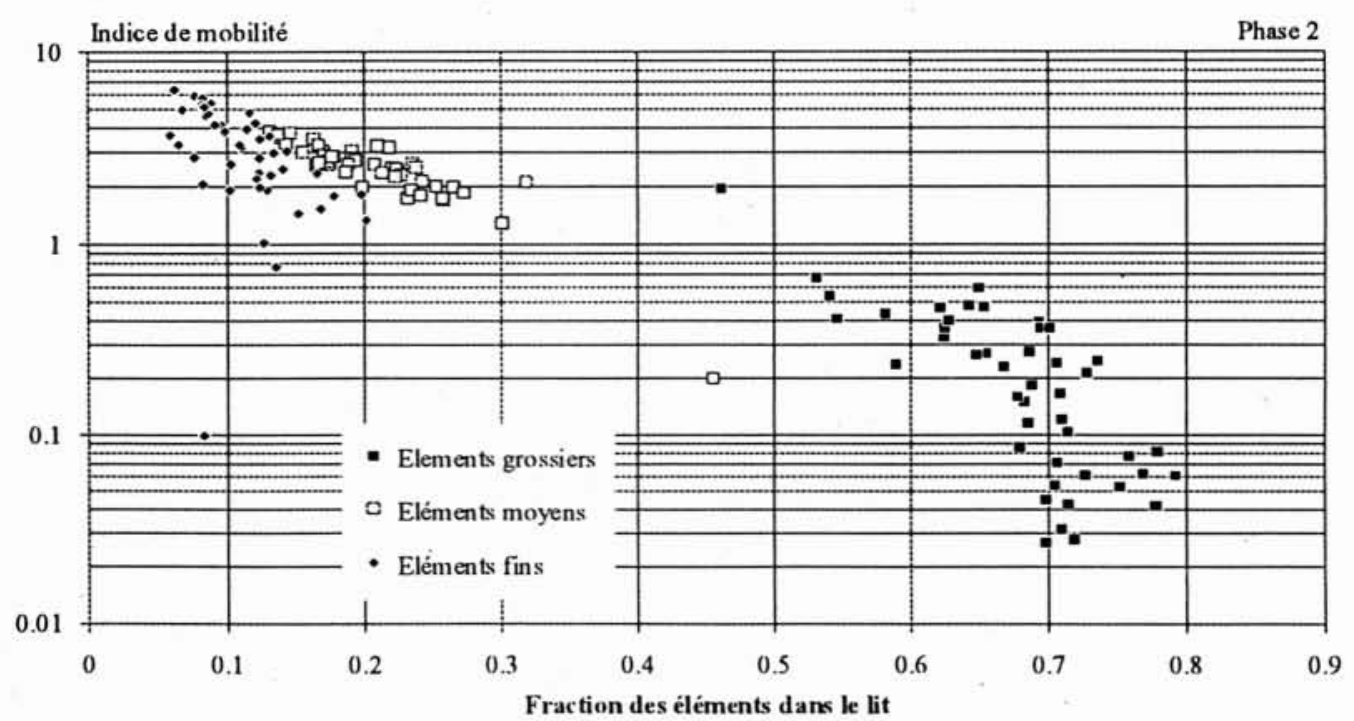

16. Indice de mobilité des éléments en fonction de leur fraction dans le lit durant la deuxième phase.

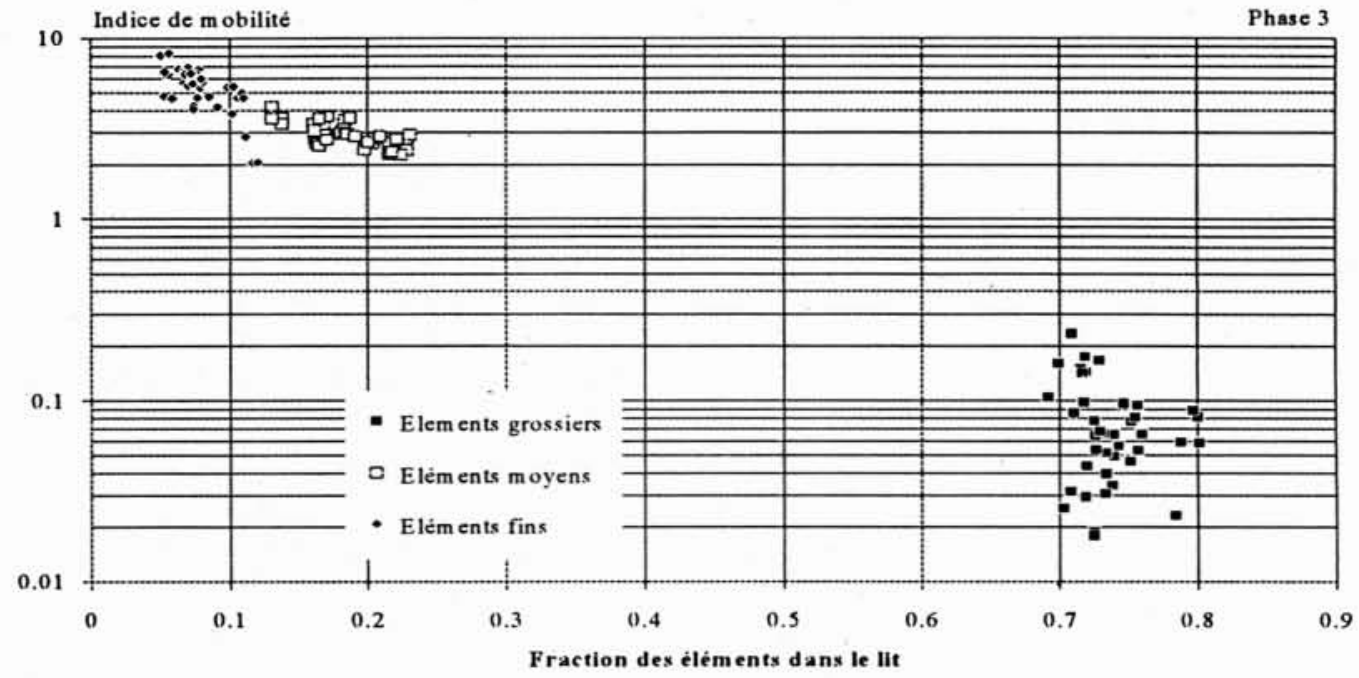

17. Indice de mobilité des éléments en fonction de leur fraction dans le lit durant la troisième phase. 


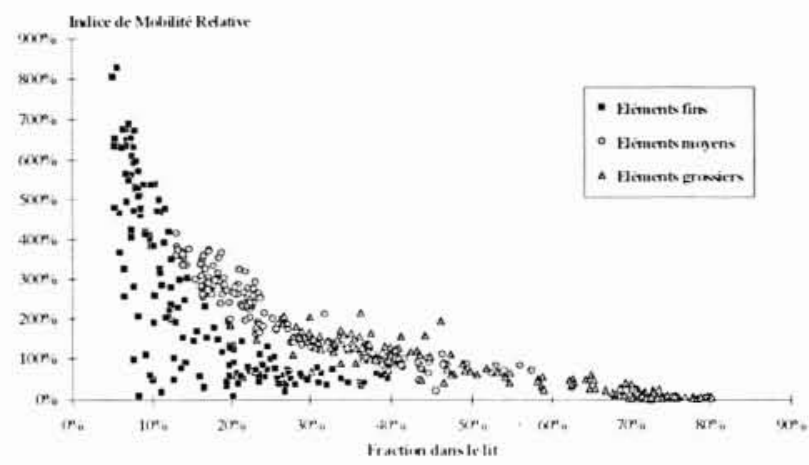

18. Mobilité de chaque classe granulométrique en fonction de sa fraction dans le lit pour liensemble des essais.

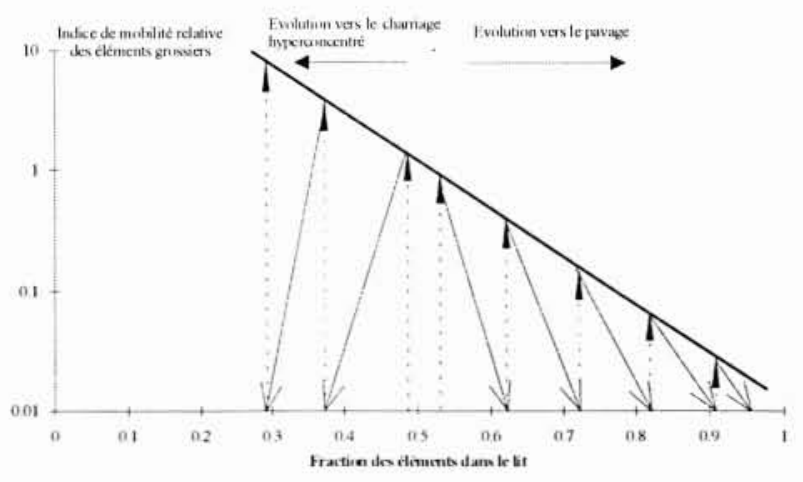

19. Schéma dévolution de la granulométrie du lit à partir de l'indice de mobilité relative.

granulométriques dans le lit pour des conditions hydrauliques et de transport solide très différentes:

L'indice de mobilité relative permet de mieux comprendre le caractère instationnaire des processus. En effet, il apparaît, comme le montre la figure 19, que la mobilité d'une classe granulométrique est d'autant plus grande que cette classe est peu représentée dans le lit.

En conséquence, toute évolution d'une classe granulométrique a tendance à s'amplifier, ce qui entraine une forte instabilité du phénomène du tri granulométrique lorsque les conditions extérieures (apport amont, pente, débit liquide) ne varient pas.

Le schéma est le suivant (en considérant par exemple les éléments grossiers) :

- Si l'on part d'une forte présence d'éléments grossiers, celle des éléments moyens et fins est faible. L'arrachement des grains dans le lit est alors plus important pour les éléments fins et moyens que pour les éléments grossiers. - On obtient, à l'étape suivante, une fraction encore plus grande d'éléments grossiers et encore plus faible d'éléments moyens et fins.

- L'écart de mobilité se creuse encore et le phénomène s'emballe. Cependant, comme il correspond à une diminution du débit solide, le lit devient pavé et stable.

De même, en partant d'une fraction grossière faible, la mobilité des éléments grossiers est forte et l'on obtient par la suite un lit encore plus fin. On se trouve alors dans le cas du charriage hyperconcentré qui présente - localement - un équilibre stable car imposé par les conditions hydrauliques. Cet équilibre correspond à un très fort transport solide. C'est le cas du chenal central des nappes de charriage hyperconcentré.

Ainsi, le tri granulométrique présente une forte instabilité, dans le cas du charriage torrentiel, lorsque le pavage commence. Or, toutes les classes connaissant la même tendance (augmentation de la mobilité avec la diminution de la fraction dans le lit), aucun élément régulateur interne n'existe dans le tri granulométrique.

Le tri granulométrique peut conduire à deux situations diamétralement opposées (pavage ou charriage hyperconcentré). On a alors un processus de bifurcation avec une évolution irréversible vers une situation stable.

Cependant, les conditions extérieures (pente, apports amonts, débit liquide) régulent les phénomènes et l'instabilité ne se produit que dans une plage réduite, lorsque l'arrêt durable des éléments grossiers est possible mais non systématique.

Lorsque le transport solide amont est important, le tri granulométrique peut amorcer localement une évolution vers le pavage ; les conditions extérieures imposent alors la réversibilité en bouleversant localement le système lit + écoulement. En effet, un amas de gros matériaux peut faciliter l'arrêt d'autres grains grossiers, mais cet amas ne reste immobile que temporairement: par sapement de l'aval, il est vite remis en mouvement et génère une bouffée de transport solide.

Il semble possible, sur la base des résultats obtenus jusqu'ici, de construire un modèle décrivant l'évolution locale du lit et du transport solide dans un lit torrentiel. Pour ce faire, il a fallu scinder le problème en deux :

- Le calcul du débit solide global. Il s'agit d'un problème ardu et qui nécessite probablement de prendre en compte le volume de matériaux mobilisables dans le lit.

- La détermination du tri granulométrique. Cette modélisation a été réalisée à partir des essais précédents. Elle montre le caractère très instable à la fin de la phase de très fort transport solide. En effet, le modèle converge très rapidement, soit vers un lit pavé, soit vers un lit très lisse correspondant au charriage hyperconcentré.

Cependant, il semble possible de construire un modèle complet des phénomènes ponctuels, en élargissant la démarche déjä employée. Il reste encore à prendre en compte les éléments suivants :

- Détermination du débit solide.

- Prise en compte des interactions conditions hydrauliques $<=>$ lit torrentiel.

- Influence du transport solide provenant de l'amont (débit solide mais surtout granulométrie du matériau transporté).

Il s'agit d'un objectif à long terme, mais il semble qu'on aurait alors un moyen puissant de comprendre, puis éventuellement de prévoir, le comportement d'un torrent, en élargissant le calcul ponctuel à toute une zone au moyen d'un maillage. Cependant, il est encore nécessaire, avant de construire le modèle numérique, de se doter des équations 
de base de ces phénomènes à partir de mesures sur canal "ponctuel".

\section{Analyse du tri granulométrique en canal large}

L'essai en canal large (essai bidimensionnel) est destiné à comparer un écoulement libre de divaguer dans ces propres alluvions, à l'écoulement en canal étroit à une seule dimension en plan (essai monodimensionnel).

\subsection{Discussion du protocole d'essai}

Le protocole d'essai adopté pour le canal large $(0,6 \mathrm{~m})$ est aussi semblable que possible au protocole de l'essai en canal étroit. Les trois étapes, à savoir transport intense avec apport amont, pavage et dépavage (sans apport) ont été maintenues.

Toutefois, on a observé que le débit liquide assurant la mise en mouvement est plus élevé (le débit minimum est de $0,31 / \mathrm{s}$ au lieu de $0,2 \mathrm{l} / \mathrm{s}$ ).

Mais deux différences essentielles doivent être soulignées avant présentation des résultats :

- Avec l'essai bidimensionnel, la technique du calcul de la granulométrie en place par l'estimation de la couche active est impossible car la localisation et la superficie de la couche active varient avec le temps. On ne peut donc plus estimer la granulométrie du lit pendant l'essai.

- Si les paramètres de la première étape de l'essai sont semblables pour les deux configurations (apport solide amont et fort transport solide), l'évolution des fonds durant cette première étape est très différente :

- Le contrôle de l'égalité des débits solides, entrant et sortant, est, pour le canal large, très grossier: le stock alluvionnaire du modèle est en effet important.

- Au contraire de ce qui se passe en monodimensionnel, le matériau dans le canal large subit une modification de sa granulométrie durant la première phase distincte de la modification observée dans la deuxième étape. En effet, si la granulométrie introduite en amont du modèle est identique à la granulométrie du modèle au début de l'essai, la granulométrie sortante est différente. On peut donc dire que lors de la première étape, on n'a atteint ni la «permanence » du débit solide, ni la "permanence» de la

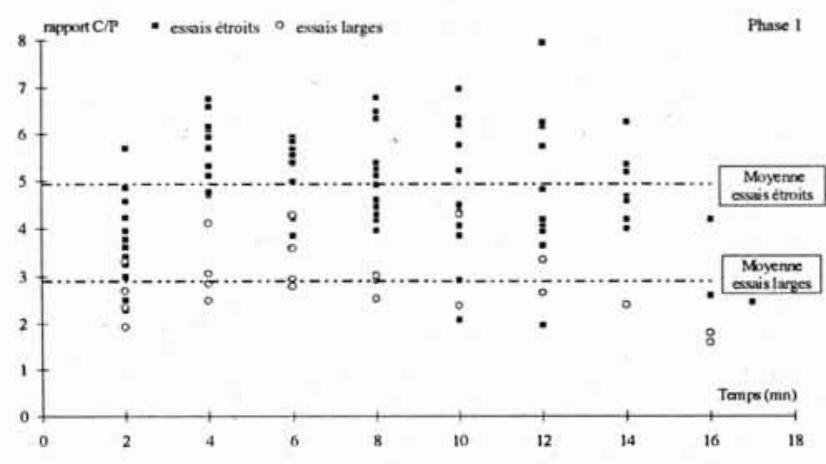

20. Comparaison du rapport $C / I$ des essais en canal de $10 \mathrm{~cm}$ de large avec ceux en canal de $60 \mathrm{~cm}$ de large.

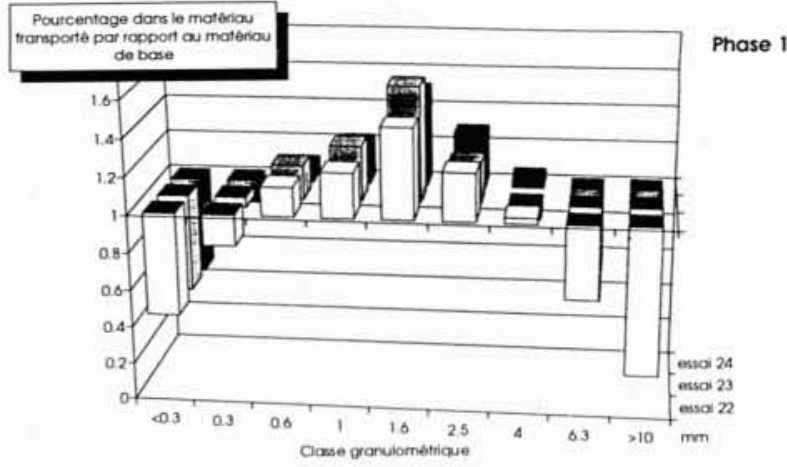

21. Débits liquide et solide en fonction du temps.

granulométrie sortante: le processus de tri lors de la deuxième étape peut s'en trouver modifié. Il n'en est pas de même lors de l'essai monodimensionnel, par suite de la faiblesse des volumes mis en jeu et de l'importance du débit solide initial.

Cette observation suggère, pour les travaux futurs, la mise en œuvre d'un protocole d'essai différent. Au cours de la première étape, le matériau serait recyclé en circuit fermé: le volume sortant serait, avec sa granulométrie propre, immédiatement réinjecté en amont du modèle : après stabilisation (globale) du lit (débits liquide et solide, pente, granulométrie), le circuit sera ouvert et le processus de pavage testé pour différents débits liquides.

\subsection{Résultats obtenus}

\subsubsection{Capacité de transport lors de la première étape des essais}

On observe, au cours de la première phase, comme le montre la figure 20, que la concentration Qs/Ql était de l'ordre de 1,5 à 2 fois plus faible dans le canal large par rapport au canal étroit. Cette constatation n'est pas surprenante car l'écoulement ayant la possibilité de divaguer, le débit liquide de début d'entraînement était plus élevé en canal large qu'en canal étroit.

Cela pose le problème d'une éventuelle différenciation de l'emploi des formules de transport solide suivant qu'elles ont été obtenues à partir d'essais en canal étroit (largeur contrainte) ou large (largeur ajustée par l'équilibre dynamique). Mais surtout, cela renvoie au problème de la détermination de la largeur limite qui sépare les écoulements libres des écoulements contraints.

\subsubsection{Granulométrie du matériau transporté}

La plus grosse partie du matériau transporté en aval du modèle provient de la première étape de $10 \mathrm{~min}$ avec fort transport solide.

Durant cette étape, nous avons comparé la composition du matériau de base et du matériau sortant.

Il apparaît que le matériau transporté a une composition en matériaux fins et grossiers plus faible que le matériau en place ; s'il faut formuler des réserves pour la validité de la mesure des fines, qui peuvent avoir échappé à la mesure, on ne peut écarter totalement la possibilité de stockage 


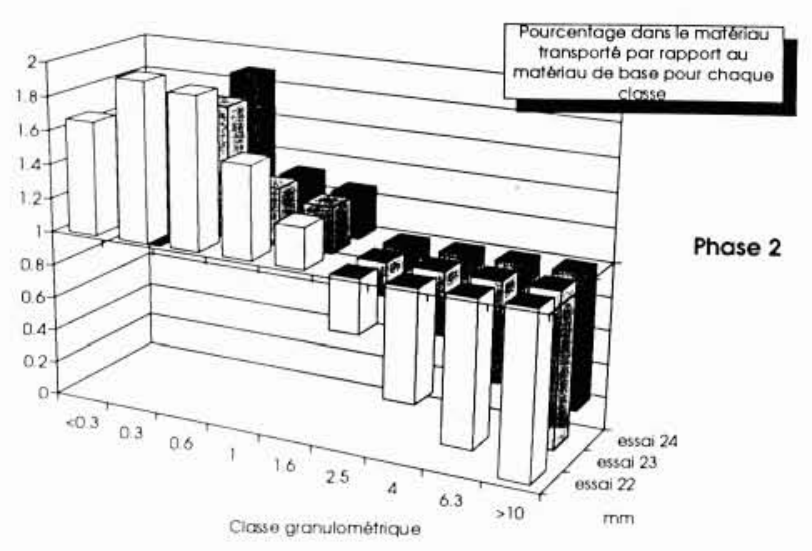

22. Evolution du débit liquide de dépavage suivant les essais et les critères retenus.

d'éléments fins dans les structures morphologiques qui se forment sur le lit durant cette phase (stockage de matériaux fins dans une mouille par exemple). D'autre part, la proportion des deux classes supérieures est la moitié de celle du matériau de base. Ce constat met bien en évidence la modification du lit durant la première étape.

En outre, on constate que, d'un essai à l'autre, chaque classe granulométrique est transportée dans les mêmes proportions bien que le volume sortant ait varié de 9 à 14 litres.

La même analyse du volume sortant peut être faite pour la deuxième étape, à savoir la formation du pavage, pour des débits permanents de 0,3 à $0,41 / \mathrm{s}$ :

Deux constatations s'imposent:

- Dans la phase de décroissance du transport solide qui conduit à l'arrêt, la décroissance granulométrique est considérable et conforme à celle de l'essai monodimensionnel.

- D'un essai à l'autre, la distribution granulométrique du volume sortant est liée au débit liquide de l'essai. Ainsi, si on isole les trois classes granulométriques supérieures, on voit qu'elles correspondent à :

$6,5 \%$ pour l'essai 22 (débit liquide $0,31 / \mathrm{s}$ ),

$16,2 \%$ pour l'essai 24 (débit liquide $0,4 \mathrm{l} / \mathrm{s}$ ).

Plus précisément, on peut calculer les volumes apportés au cours de l'étape du pavage pour chaque essai en distinguant :

- Les matériaux d'un diamètre supérieur $4 \mathrm{~mm}$,

- Les matériaux de diamètre médian,

- Les matériaux d'un diamètre inférieur à $1,6 \mathrm{~mm}$.

\begin{tabular}{|l|c|c|c|c|c|}
\hline \multicolumn{2}{|c|}{} & \multicolumn{3}{c|}{ Volume par classe } \\
\hline Essai & $\begin{array}{c}\text { Débit liquide } \\
(1 / \mathrm{s})\end{array}$ & $\begin{array}{c}\text { Volume } \\
\left(\mathrm{cm}^{3}\right)\end{array}$ & $>4 \mathrm{~mm}$ & médian & $<1.6 \mathrm{~mm}$ \\
\hline 22 & 0.3 & 1460 & 96 & 322 & 1042 \\
23 & 0.36 & 3850 & 565 & 936 & 2349 \\
24 & 0.4 & 6100 & 986 & 1397 & 3717 \\
\hline
\end{tabular}

On voit qu'il n'y a pas de différence fondamentale dans la répartition granulométrique des éléments transportés. Par contre, le volume des matériaux grossiers déplacés est plus de 10 fois supérieur dans le cas de l'essai 24 : il semble donc qu'un seuil ait été franchi et que, lorsque l'écoulement devient capable de déplacer des éléments grossiers, il peut en transporter beaucoup et dans des proportions importantes. La formation du pavage se fait au voisinage du seuil de début de transport des matériaux du lit (avant pavage bien sûr!).

\subsection{Conclusion}

En conclusion, l'équilibre dynamique d'un lit à deux dimensions peut être traduit ainsi : quand la largeur est libre de se fixer en équilibre avec l'écoulement, elle s'adapte à une valeur pour laquelle le rapport $h / d_{90}$ est de l'ordre de l'unité, le nombre de Froude est proche de la valeur critique, et la contrainte de cisaillement avoisine la valeur de début de mouvement. On est donc placé dans un " espace de forte variabilité » de toutes les grandeurs interdépendantes, dont la détermination fine devient illusoire.

Cette liberté supplémentaire s'ajoute aux phénomènes observés en canal étroit. Elle est susceptible de modifier totalement le comportement de l'ensemble lit + écoulement. Ainsi, les essais de dépavage conduits en canal de $60 \mathrm{~cm}$ de large ont montré que celui-ci n'a plus du tout la même réalité que dans le cadre des essais monodimensionnels: il ne s'agit plus de l'arrachement des grains du fond, mais d'un prélèvement latéral.

On retrouve dans ces essais la notion de débit de plein bord utilisée par les géomorphologues. En effet, le lit ne commence à changer que lorsque le lit principal écoule un débit suffisant pour qu'une partie de l'écoulement passe dans des bras secondaires. Il semble donc que le débit de plein bord corresponde à la limite de stabilité dans un tel cas.

Ainsi, les phénomènes de divagations se traduisent par l'apparition de nouveaux phénomènes, quelquefois contradictoires - en apparence - avec les observations réalisées dans le cadre d'un écoulement monodimensionnel. Il devient alors nécessaire de ne pas limiter l'étude du transport solide aux écoulements en canal étroit.

\section{Bibliographie}

[1] Mizuyama T. (1977). - Bedload transport in steep channels. $\mathrm{Ph}$. D Dissertation, Kyoto Univ., Japan. $118 \mathrm{p}$.

[2] SMART G.M., JAEGGi M. (1983). - Sediment transport on steep slopes. Zurich. Mitteilung der Versuchsanstalt fur Wasserbau, Hydrologie und Glaziologie, ETH N ${ }^{\circ} 64,191$ p.

[3] Rickenmann D. (1990). - Bedload transport capacity of slurry flows at steep slopes. Versuchsanstalt für Wasserbau, Hydrologie und Glaziologie der Eidgenössichen Technischen Hochschule Zürich. Mitteilungen 103. 249 p.

[4] MEunier M. (1991). - Eléments d'hydraulique torrentielle. CEMAGREF Série Etudes Montagne $\mathrm{N}^{\circ}$ 1. 278 pages.

[5] TAKAHASHI T. (1987). - High velocity flow in steep erodible channels. XII ${ }^{e}$ Congrès AIRH Lausanne.

[6] GRAF W.H. (1989). - Flow resistance over a gravel bed; its consequence on initial sediment movement. International 
Workshop on fluvial hydraulics of mountain rivers. Trent. Al-A17.

[7] RiCKenMANn D. (1990). - Debris flow 1987 in Switzerland: modelling and fluvial sediment transport. Symposium on Hydrology in Mountainous regions. AIHS Publ. N 194. Vol. 2.

[8] LISLE T.E. (1987). - Channel morphology and sediment transport in steepland streams. Erosion and sediment in the Pacific Rim-AIHS Publ. N`165. Corvallis. August 1987.

[9] Meunier M. et CARION C. (1987). - Etude méthodologique de la détermination des courbes gramulométriques des lits des cours d'eau CEMAGREF. Grenoble. $32 \mathrm{p}$.

[10] SIMON L. (1986). - Transport solide de sédiments de granulométrie non uniforme. Laboratoire National d'Hydraulique de Chatou. EDF.

[11] Rahuel J.L. (1988), - Modélisation de l'évolution du lit des rivières alluvionnaires à granulométrie étendue. Thèse INPG. Grenoble. 248 p.

[12] DUVERT C. (1993). - Contribution à l'étude du pavage.
CEMAGREF-ENITRTS (Mémoire de 3 année).

[13] Koulinski V. (1993). - Etude de la dynamique d'un lit torrentiel par confrontation d'essais sur modèle réduit et d'observations de terrain. Thèse UJF-CEMAGREF-SOGREAH (soutenance en automne 1993).

[14] Displas P. (1986). - Bed load transport in gravel-bed streams : some properties. Proceedings 3rd Intern. Symp. on River Sedimentation. Jackson. Mississipi.

[15] ANDREWS E.D. (1983). - Entrainment of gravel from naturally sorted riverbed material. Geol. Soc. Am. Bull. N94.

[16] Parker G., Klingeman P.C. (1982). - On why gravel bed streams are paved. J. of Water Resources Research, Vol. 18 , $\mathrm{N}^{\circ} 5$.

[17] BATHURST J.C. (1987). - Critical conditions for bed material movement in steep, boulder-bed streams». Corvallis Symposium : Erosion and sedimentation in the Pacific Rim. AIHS Publ. $\mathrm{N}^{\circ} 165$.

[18] EglaZAROFF I.V. (1965). Calculation of nonuniform sediment concentrations. Proc. ASCE, Vol.91, HY4. 